\title{
Light-sheet Bayesian microscopy enables deep- cell super-resolution imaging of heterochromatin in live human embryonic stem cells
}

\author{
Ying S Hu${ }^{1 \dagger}$, Quan Zhu ${ }^{2^{*}}$, Keri Elkins ${ }^{2,4}$, Kevin Tse $^{3}, \mathrm{Yu} \mathrm{Li}^{1}$, James A J Fitzpatrick', Inder M Verma ${ }^{2^{*}}$ and Hu Cang ${ }^{1^{*}}$
}

\begin{abstract}
Background: Heterochromatin in the nucleus of human embryonic cells plays an important role in the epigenetic regulation of gene expression. The architecture of heterochromatin and its dynamic organization remain elusive because of the lack of fast and high-resolution deep-cell imaging tools. We enable this task by advancing instrumental and algorithmic implementation of the localization-based super-resolution technique.

Results: We present light-sheet Bayesian super-resolution microscopy (LSBM). We adapt light-sheet illumination for super-resolution imaging by using a novel prism-coupled condenser design to illuminate a thin slice of the nucleus with high signal-to-noise ratio. Coupled with a Bayesian algorithm that resolves overlapping fluorophores from high-density areas, we show, for the first time, nanoscopic features of the heterochromatin structure in both fixed and live human embryonic stem cells. The enhanced temporal resolution allows capturing the dynamic change of heterochromatin with a lateral resolution of $50-60 \mathrm{~nm}$ on a time scale of $2.3 \mathrm{~s}$.

Conclusion: Light-sheet Bayesian microscopy opens up broad new possibilities of probing nanometer-scale nuclear structures and real-time sub-cellular processes and other previously difficult-to-access intracellular regions of living cells at the single-molecule, and single cell level.
\end{abstract}

Keywords: Super-resolution imaging; Light sheet; Bayesian; Heterochromatin; Human embryonic stem cell

\section{Background}

In the nucleus of eukaryotic cells, chromatin is organized into two distinct domains: the lightly packed and actively transcribed euchromatin, and the condensed and transcriptionally silent heterochromatin. Although transcriptionally repressive, heterochromatin is known to provide binding sites for regulatory proteins, and therefore, plays an important role in the epigenetic regulation of a variety of biological processes, such as development, cellular differentiation and maintenance of genome integrity (Maison \& Almouzni 2004). Human embryonic stem cells (hESCs) are among the most commonly utilized pluripotent cells that hold promise in tissue replacement

\footnotetext{
* Correspondence: qzhu@salk.edu; verma@salk.edu; hucang@salk.edu ${ }^{\dagger}$ Equal contributors

${ }^{2}$ Laboratory of Genetics, Salk Institute for Biological Studies, La Jolla, CA 92037, USA

${ }^{1}$ Waitt Advanced Biophotonics Center, Salk Institute for Biological Studies, La Jolla, CA 92037, USA

Full list of author information is available at the end of the article
}

therapy for human diseases. Although hESCs are well characterized as being globally hyperactive in transcriptional activities, better characterization of the structure and dynamics of heterochromatin promotes in-depth understanding of their unique developmental potential which gives rise to different cell lineages, such as bone, muscle, and neural cells (Thomson et al. 1998). Recent developments in high-throughput DNA sequencing-based chromatin conformation capture assays suggest complex topographical organization of megabase-sized domain structures in the genome architecture (Dixon et al. 2012; Lieberman-Aiden et al. 2009). However, the sequencing techniques only provide averaged topological information from an ensemble of cells. Direct visualization of these domain structures in individual cells is a key step to advance the epigenetic study by bridging the genome architecture with the underlying regulatory processes. A viable imaging technique with high spatial and temporal resolution will fulfill this role.

Single-molecule super-resolution imaging is capable of extracting detailed structural information from biological 
samples in a physiologically viable environment and achieving a spatial resolution on par with the electron micrograph (Rust et al. 2006; Betzig et al. 2006; Hess et al. 2006). Key to achieving a high spatial resolution is a high signal-noise ratio (SNR) and a large number of identifiable single molecule events. On one hand, the localization width of a single-molecule event is inversely related to its SNR. On the other hand, because localizations are identified from a set of acquired images, traditional PALM and STORM techniques rely on thousands of individual imaging frames to achieve a lateral resolution of a few tens of nanometers. The acquisition typically takes several minutes, and has largely hindered this promising technique from gaining more popularity and providing mechanistic insights to biomedical studies.

The thickness of hESCs poses a major challenge to achieve high SNR with conventional imaging modalities (Patterson et al. 2010; Moerner 2007; Rust et al. 2006; Betzig et al. 2006; Hess et al. 2006). Individual hESCs have a large cell volume (Zwaka \& Thomson 2003) and a nucleus that is thicker than $10 \mu \mathrm{m}$ (Additional file 1: Figure S1). Total-internal-reflection microscopy (TIRF) is a widely adapted high-SNR technique for super-resolution imaging. However, TIRF has a limited probing depth of $\sim 200 \mathrm{~nm}$, covering only the basal membrane of a cell (Huang et al. 2008; Bates et al. 2007; Lippincott-Schwartz \& Patterson 2009). Epi-illumination can reach deeper inside a cell at the cost of exciting unwanted molecules and poor SNR due to the generation of out-of-focus fluorescence. We resort to a selective-plane illumination strategy, which has been used for tissue-level imaging (Ahrens et al. 2013; Keller et al. 2010; Keller et al. 2008; Tomer et al. 2012) and single molecule tracking (Ritter et al. 2010; Friedrich et al. 2009), to improve the background for super-resolution imaging of thick samples.

Importantly, imaging live hESCs also requires a much faster imaging speed to counter cell motion and to capture dynamic changes that cannot be obtained by any other biotechnological means. Standard single-emitter fitting programs render the technique impractically slow (i.e. minute-scale acquisition times), even with the aid of the light-sheet illumination. Since the Nyquist sampling theorem requires approximately two data points per resolution unit to faithfully reconstruct an image (Shroff et al. 2008; Patterson et al. 2010), reducing the number of image frames requires increasing the number of resolvable single-molecule events per frame. Spatially overlapping molecules within a diffraction-limited focus volume in the same frame breaches the sparcity requirement of many singleemitter fitting programs (Henriques et al. 2010; Wolter et al. 2012) and further slow down the data acquisition. Here, we demonstrate a high-SNR illumination scheme that works hand in hand with a highly efficient reconstruction algorithm resolving overlapping fluorophores to enable rapid deep-cell super-resolution imaging.

\section{Methods}

Optical design for prism-coupled light-sheet illumination

To reject out-of-focus fluorescence background from thick samples, selective-plane illumination microscopy (SPIM), also known as light-sheet microscopy, has been recently introduced for single-molecule super-resolution imaging by Zanacchi et al (Cella Zanacchi et al. 2011). The planar illumination is wide-field compatible and faster than scanning-based two-photon techniques for large areas (Folling et al. 2007; York et al. 2011; Vaziri et al. 2008). To generate a light sheet, laser light passes through a cylindrical lens and focuses to a line onto the back aperture of an illumination objective, which delivers the planar sheet of light to an imaging sample (Figure 1a). For imaging the intact nucleus with high speeds, light-sheet illumination provides optical sectioning with ease. While the concept of SPIM for single-molecule imaging has been successfully demonstrated, the original light-sheet condenser design has essentially remained unchanged since its conception by Siedentpf and Zsigmondy in 1903 (Siedentpf and Zsigmondy 1903). Considerable efforts have been devoted to searching for a method to induce light sheet illumination suitable for sectioning sub-cellular regions, while accommodating the use of a high-numerical-aperture $(\mathrm{NA} \geq 1.0)$ objective lens for high photon collection efficiency, and providing a large and flat field of view (FOV) to cover the entire region of interest. For example, the individual molecule localization SPIM (IML-SPIM) (Cella Zanacchi et al. 2011) and inverted SPIM (iSPIM) (Wu et al. 2011) (Figure 1b-c) impose a space constraint to simultaneously support a thin light sheet and a high-NA collection objective from the orthogonally oriented objectives. Alternatively, highly inclined and laminated optical sheet (HILO) uses a single high-NA objective; but its FOV is limited due to the oblique illumination (Tokunaga et al. 2008) (Figure 1d). A recent design by Gebhardt et al. attempted to address this problem by using a polished AFM cantilever as a mirror, which was placed close to the sample (Figure 1e). The mirror deflects the light sheet from the vertically-oriented illumination objective to generate a horizontally-oriented illumination plane coupled to a high-NA imaging objective with a slight horizontal offset on an inverted microscope (Gebhardt et al. 2013). This technique induces a $2-\mu \mathrm{m}$ gap that cannot be imaged in the samples.

To adapt the light sheet illumination for imaging single hESCs, we have developed a simple and effective prismcoupled light-sheet condenser design. Demonstrated in Figure 1f and Additional file 2: Figure S2, light sheet was created by an $f=500 \mathrm{~mm}$ plano-convex cylindrical lens (Thorlabs LJ1144RM-A) and focused onto the back focal 


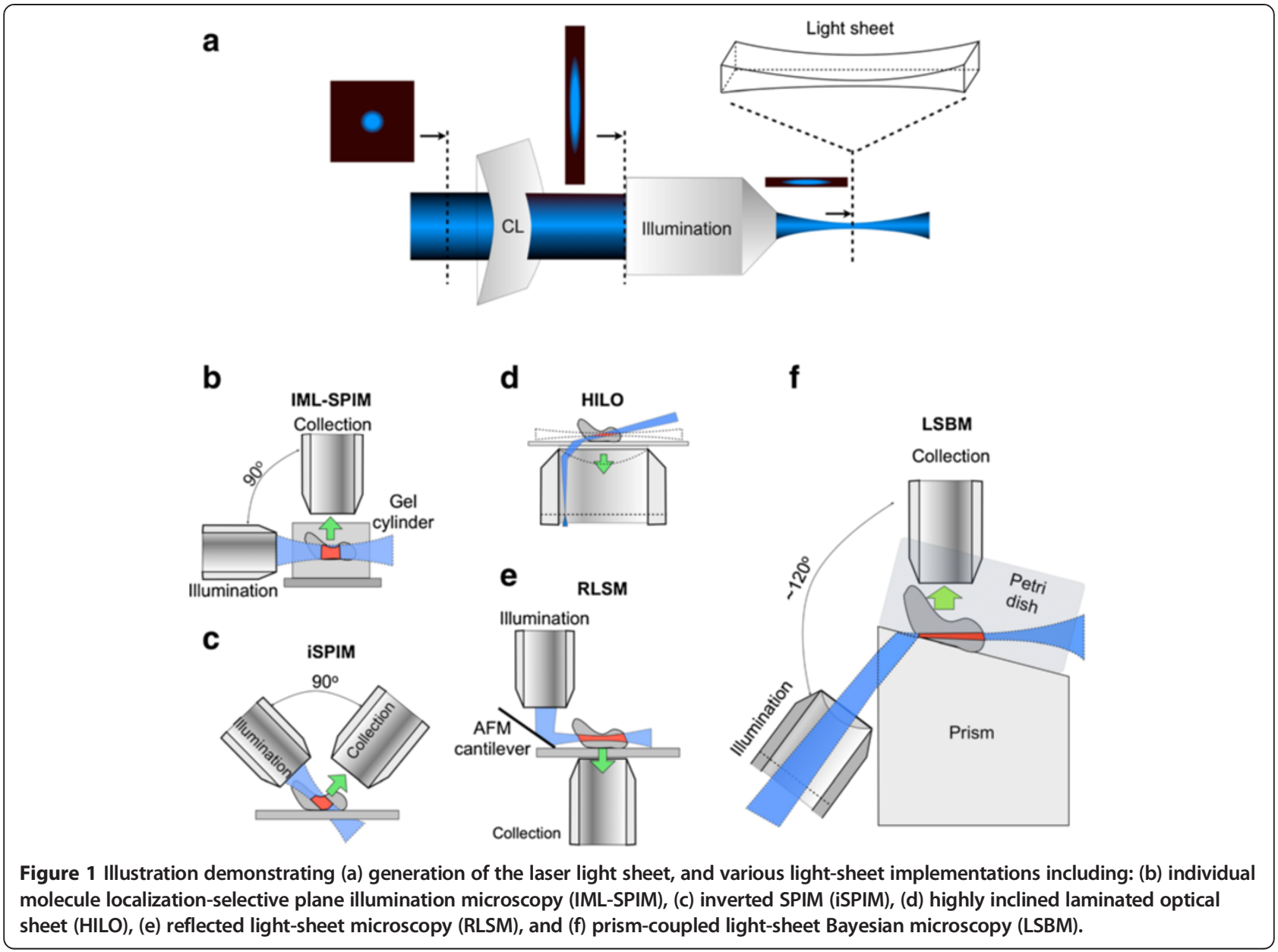

plane of an infinity-corrected Mitutoto M Plan APO 50x NA 0.55 condenser objective. A Pellin-Broca prism (Thorlabs ADBU-10) was placed after the condenser objective to redirect the light sheet horizontally onto a sample at the focal plane of an imaging objective, in our case, a Zeiss W Plan-Apochromat 63x NA 1.0 water immersion lens.

The prism-coupled condenser design enjoys three major advantages for super-resolution imaging. First, the de-coupling between the imaging and condenser objective and increase of the angle between them from 90 to roughly 120 degrees relaxes the space constraint for sample placement. The design widens the choice of imaging objectives and allows the use of even higher NA (NA > 1.0) water-immersion lenses. Second, the horizontal overlap between light-sheet illumination and the imaging plane yields a flat and large FOV with high SNR. Large FOV is necessary in order to capture the entire cross-section of the nucleus of the hESC. Third, compared to the AFM cantilever system (Gebhardt et al. 2013), the prism design is less prone to vibrations and simpler to implement in biology labs. In addition, the design is directly compatible with cell culture by accepting standard petri dishes. In our setup, we used a 35-mm glass-bottom petri dish (MatTek P35G-0-20-C) as the sample holder. For sample placement, we custom made an aluminum adaptor plate clamped onto a piezo translation stage (Thorlabs NanoMax-TS) and tilted at a horizontal angle of approximately 20 degrees (Additional file 1: Figure S1). The adaptor held the glass bottom dish flush with the top surface of the prism with indexmatching oil in between. The piezo stage (Thorlabs NanoMax-TS) translated the sample along the top surface of the prism and exposed different depths of the hESC to light-sheet illumination. The excitation lasers, a 150-mW 561-nm diode-pumped solid-state laser (Cobolt Jive) and a 160-mW 642-nm diode laser (Coherent Cube), were brought together before the cylindrical lens and the condenser lens. Sharing the illumination path was a $60 \mathrm{~mW} 405 \mathrm{~nm}$ (Omicron PhoxX) laser for activation. For fluorescence imaging, an emission filter (600/ 60 for mEos and 705/130 for Alexa 647) and a notch filter were placed in front of an EMCCD camera (Andor iXon DU-897E with a pixel size of $16 \times 16 \mu \mathrm{m})$. For imaging fixed cells, we used a custom mounting for the 
camera with a $300-\mathrm{mm}$ tube lens, bringing the effective magnification to $115 \times$ (Additional file 2: Figure S2a). For live cell imaging, we adapted the light sheet system to the framework of a Zeiss Examiner.D1 upright microscope with mechanical support (not shown).

\section{Characterization of the light sheet}

We used the RF module in COMSOL Multiphysics ${ }^{\mathrm{TM}}$ to perform the full-wave electromagnetic simulation of the light sheet. The incident Gaussian beam was characterized in the form:

$$
\begin{aligned}
E(r, z)= & E_{0} \frac{w_{0}}{w(z)} \exp \left[-\frac{r^{2}}{w(z)^{2}}\right] \\
& \exp \left[-i\left(k z-\tan ^{-1} \frac{z}{z_{R}}+\frac{k r^{2}}{2 R(z)}\right)\right],
\end{aligned}
$$

where $r$ is the radial distance from the center of the beam, $z$ is the axial distance, $w_{0}$ is the beam waist and set to $2 \mu \mathrm{m}, w(z)=w_{0} \sqrt{1+\left(\frac{z}{\pi w_{0}^{2} / \lambda}\right)^{2}}$ is the radius at which the intensity of the beam drops to, $1 / e^{2}, R(z)=z\left[1+\left(\frac{\pi w_{0}^{2}}{\lambda z}\right)^{2}\right]$ is the radius of the wave fronts. The Gaussian wave was launched from the bottom of the 2-D simulation domain with an excitation of the magnetic field in $z$. We plotted the intensity as the square of the normal $E$ field. The FWHM from the line plot of the intensity profile characterized the width and length of the light sheet. For demonstration, we simulated the light sheet at $561 \mathrm{~nm}$. The refractive index was 1.333 for water and 1.512 for glass.

For visualizing and measuring the thickness of the light sheet, we used fluorescent bead solution (Invitrogen F8794 ) with $10^{6} \times$ dilution. A glass bottom dish containing $3 \mathrm{ml}$ diluted fluorescent bead solution was use for profiling. The thickness was determined from the image captured by the EMCCD camera and the image pixel size.

\section{Sample preparation via transgenetic labeling or immunostaining}

Human embryonic stem cells were cultured at the Stem Cell Core facility of the Salk Institute as previously described (Woods et al. 2011). Two NIH-registered hESC cell lines were cultured: WA01 (H1) and (Hues6). Both cell lines were determined to be karyotypically normal by cytogenetic analysis and shown to be pluripotent by in vivo teratoma histological assays. Pluripotent cell lines were cultured and expanded using Matrigel (BD
Biosciences, San Diego, CA) to maintain their pluripotency. To generate transgenic cell lines, we constructed lentiviral vectors, pBOB-CAG-mEos2 and pBOB-CAG$\mathrm{mEos} 3$, to express fusion proteins of mEos2-HP1 $\alpha /$ mEos3-HP1 $\alpha$ or mEos2-Centrin2/mEos3-Centrin2. Human embryonic stem cells were passaged by TrypLE digest, split into single cells, and subsequently cultured in the presence of Rock Inhibitor on Matrigel. Lentivirus infection was performed at a multiplicity of infection of $1: 20$ to 50 by spinning infection at a speed of $800 \mathrm{~g}$ for $1 \mathrm{~h}$. Transduced cells were further incubated in fresh media for $48 \mathrm{~h}$ and seeded onto glass bottom dishes.

For immunostaining, we followed standard labeling procedures for STORM. Briefly, cells were fixed in 3\% (v/v) paraformaldehyde (Alfa Aesar 43368) and 0.1\% glutaraldehyde (Sigma G5882) (v/v) in PBS for $10 \mathrm{~min}$ and washed three times in filtered PBS. The remaining PFA and glutaraldehyde were reduced by incubating fixed cells in freshly prepared 0.1\% (w/v) sodium borohydride (Sigma 213462) for $7 \mathrm{~min}$, followed by permeabilization in the blocking buffer $(5 \%(\mathrm{v} / \mathrm{v})$ normal goat serum and $0.2 \%(\mathrm{v} / \mathrm{v})$ Triton X100 (Sigma T8787) in PBS) for approximately one hour. The primary mouse anti- $\beta$-tubulin antibody (Invitrogen 1083461A) was diluted $200 \times$ in the blocking buffer with a 30-min incubation time. After washing five times in the washing buffer $(0.2 \%(\mathrm{v} / \mathrm{v})$ normal goat serum and $0.1 \%$ (v/v) Triton $\mathrm{x} 100$ in PBS), cells were incubated in the Alexa Fluor 647 conjugated goat anti-mouse IgG secondary antibody (Moleular Probes A21237) with 500× dilution in the blocking buffer for $30 \mathrm{~min}$. The sample was protected from light and post-fixed with $3 \%(\mathrm{v} / \mathrm{v})$ paraformaldehyde and $0.1 \%(\mathrm{v} / \mathrm{v})$ glutaraldehyde in PBS for $10 \mathrm{~min}$ after three-time wash.

\section{Imaging fixed and live cells}

The image frame size was cropped to smaller than $128 \times 128$ pixels in order to increase the frame rate. Low excitation power was used to first locate the nucleus or microtubules, followed by imaging with full excitation power. The initial quick bleaching and subsequent blinking process were typically captured within the first 1-2000 frames with an exposure time of 30-50 ms. Standard imaging buffer was used for STORM: $40 \mathrm{mM}$ D-glucose, 0.5 $\mathrm{mg} \mathrm{ml}^{-1}$ glucose oxidase (Sigma G6125), $40 \mu \mathrm{g} \mathrm{ml}^{-1}$ catalase (Sigma C1345), and $143 \mathrm{mM} \beta$-mercaptoethanol (Sigma M6250) at $\mathrm{pH}$ 8.0. For vertical sectioning, the sample was translated tangential to the top surface of the prism by the piezo stage. With a known tilt angle, vertical translates can be precisely determined from the tangential movement.

The light-sheet microscope was adapted for shortduration live-cell imaging. Temperature was maintained by heating the imaging objective lens and glass prism using objective heaters (MTC-HLS-025/TC-HLS-025, 
Bioscience Tools, San Diego, CA). The imaging duration was less than $30 \mathrm{~min}$. Transgenic Hues6 cells expressing $\mathrm{mEos} 3-\mathrm{HP} 1 \alpha$ were seeded on glass bottom dishes with phenol-red free TeSR 30 min prior to imaging. For 250 $\mathrm{ml}$ of colorless TeSR, $500 \mu \mathrm{l}$ of FGF-2, and $30 \mu \mathrm{l}$ of TGFb1 were added to prevent cell differentiation. To minimize adverse phototoxicity, $1 \mathrm{~mW} 405 \mathrm{~nm}$ laser was used to activate mEos3 for $5 \mathrm{~s}$, followed by bleach-andblink acquisition using the 561-nm laser at $40 \mathrm{~mW}$ for $\sim 23 \mathrm{~s}$ with 4000 frames. Approximately $26 \mathrm{~mW}$ from the $561-\mathrm{nm}$ laser and $200 \mu \mathrm{W}$ from the $450-\mathrm{nm}$ laser were delivered to the back aperture of the light sheet condenser objective. The exposure time was $50 \mathrm{~ms}$ and the frame size was $75 \times 89$ pixels. For imaging fixed cells, we used a custom-designed system and paired a 300$\mathrm{mm}$ tube lens with a $63 \times$ Zeiss objective. The effective magnification was $114 x$, yielding a pixel size of $135 \mathrm{~nm}$. For live cell imaging, we coupled light sheet into a commercial Ziess microscope with a $63 \times$ magnification, yielding a pixel size of $254 \mathrm{~nm}$. A $2.5 \times$ magnifier can be used to further reduce the pixel size to $102 \mathrm{~nm}$.

\section{Bayesian bleach-and-blink (3B) image reconstruction}

The Bayesian method developed by Cox et al. (Cox et al. 2012; Rosten et al. 2013) globally analyzes sequential frames and applies a statistical model to infer the best possible distribution of molecules using their blinking and bleaching properties across different frames. Spatially overlapping fluorophores are allowed in any particular frames and molecules are resolved by a priori knowledge about the proceeding frames and a posteriori estimates of the bleaching and blinking of the molecules in the subsequent frames. Termed $3 \mathrm{~B}$ for Bayesian bleaching and blinking analysis, the method harnesses single molecules from high-density areas and substantially shifts the burden from data acquisition to post-processing (Lidke 2012). Thorough discussion of the Bayesian theory and models of the bleaching and blinking properties of the molecules can be found in the literature (Cox et al. 2012; Rosten et al. 2013).

Prior to Bayesian analysis, we collected a set of standard STORM data using light-sheet illumination and analyzed the brightness and size distribution of the single-molecule events. The Bayesian code assumes a log-normal distribution, which closely characterizes our identified brightness $\left(R^{2}=0.9982\right)$ and size $\left(R^{2}=0.9957\right)$ values of the single-molecule events (Additional file 3 : Figure S3). In comparison to the exponential distribution commonly used for single-molecule analysis, the observed log-normal distribution is attributed by the thresholding in localization analysis and the fact that brightness and lifetime measurements are frame-rate limited and typically do not cover the entire lifespan of the molecule.
We ported the Bayesian bleach-and-blink code to a supercomputing cluster Hopper at the National Energy Research Scientific Computing (NERSC) Center at the Lawrence Berkeley National Laboratory. For image reconstruction, over-exposed images at the beginning of the acquisition were discarded and subsequent $400 \mathrm{im}$ ages (saved as fits files) were selected for processing. The $3 \mathrm{~B}$ analysis was parallelized by dividing the image into 10-by-10-pixel sub-masks, each with a $50 \%$ horizontal and vertical overlap with its neighbors to eliminate edge artifacts. A Python-MPI wrapper was used to distribute the Bayesian calculation to a number of processors equal to the number of sub- masks. For a 128by-128-pixel image, approximately 2,500 CPU hours were consumed. Details of the Bayesian algorithm and its implementation are discussed in Additional file 4: Note S1. We also provided an alternative computing resource openly distributed via Amazon and using its Elastic Cloud Computing (EC2) service for labs without the access to supercomputing resources (Hu et al. 2013).

\section{Results and discussion}

\section{SNR enhancement}

We used finite-element simulations to help tailor the design parameters for imaging the hESC nucleus, which is $\sim 10-\mu \mathrm{m}$ thick and wide. As a first-order approximation, the thickness of the light sheet is determined by the diffraction-limited waist size of a focused Gaussian beam, $w_{0}$, and its length $b$ determined by $b=2 \pi w_{0}^{2} / \lambda$. Reducing the thickness by one half shortens the length of light sheet and the effective FOV by four. We aimed to reduce the thickness of the light sheet to less than $1 / 5$ of the thickness of the nucleus while maintaining an effective range covering the entire cross-section of the nucleus. We simulated the light sheet at a 20 degree topsurface slope of the prism (Additional file 5: Figure S4) and found the thickness to be $\sim 1.1 \mu \mathrm{m}$ and length to be $\sim 14 \mu \mathrm{m}$ (Figure 2a,b). By turning the elliptical beam 90 degrees (Figure 2c), we measured the FWHM of the light sheet to be $\sim 1.8 \mu \mathrm{m}$ (Figure $2 \mathrm{~d}$ ), which is in general agreement with the calculation. The slightly wider measured profile is likely due to $3^{\text {rd }}$ order aberrations, such as coma, induced at the interface, as well as the refractive index of the imaging medium (Additional file 6: Figure S5, Additional file 7: Note S2). In addition, the finite PSF of the microscope broadens the measurements on the thickness of the light sheet at its thinnest portion. A PSF of $0.25 \mu \mathrm{m}$ of the microscope, for instance, increases the measured thickness by $0.25 \mu \mathrm{m}$. Thus, the actual thickness of the light sheet would be $\sim 1.55 \mu \mathrm{m}$.

The enhanced SNR using light sheet illumination can be observed from single-molecule images. As a comparison, we performed single-molecule imaging of fixed 

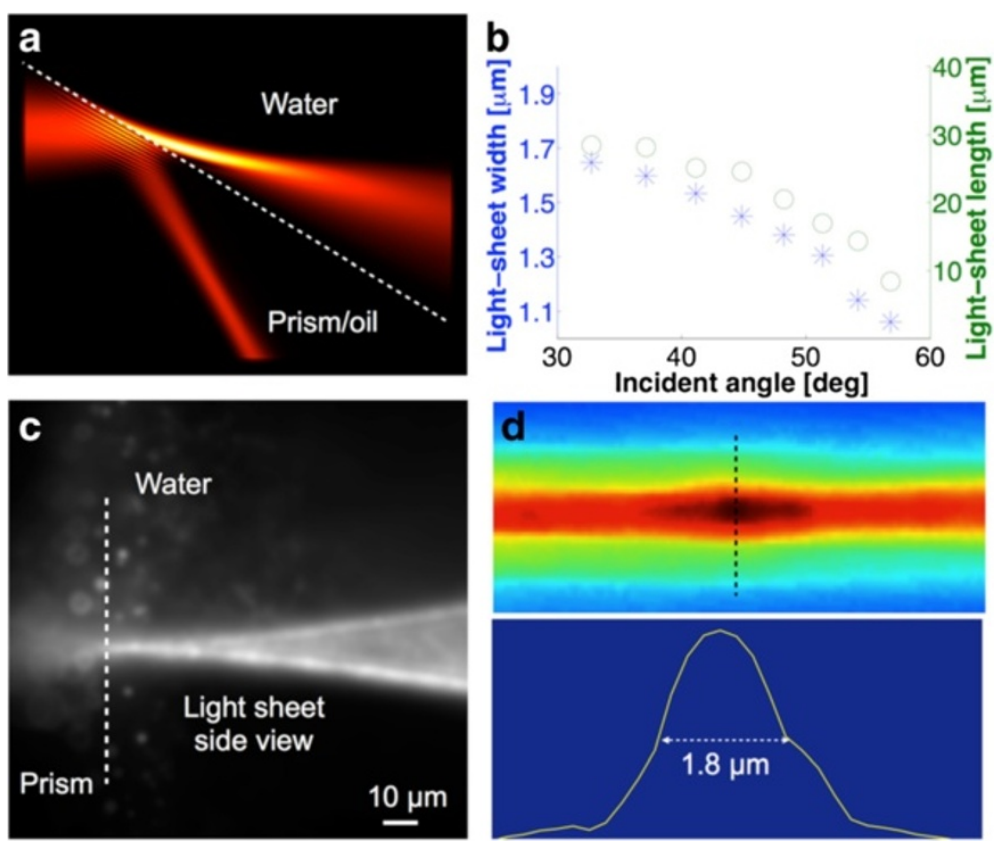

Figure 2 Characterization of the light sheet profile. (a) Electromagnetic simulation of the intensity of a focused Gaussian beam at the interface. (b) Width and length of the light sheet at various incident angles at the interface calculated from the intensity profile obtained from electromagnetic simulations. Light-sheet width was calculated from the FWHM of the intensity profile, light-sheet length was calculated from the glass-water interface along the light sheet to the point where its intensity drops by one half. Glass prism with a refractive index of 1.515 and water with a refractive index of 1.333 were used for the simulation at $561 \mathrm{~nm}$. (c) Side view of the light sheet focused at the interface where it enters water. (d) Side profile of the light sheet focused in water, i.e. further away from the interface, and the measurement of its thickness from the cross line profile.

mEos3-HP1 $\alpha$-labeled transgenic Hues6 hESCs using high-angle epi and prism-coupled light sheet illumination (Figure 3a). Figure 3b shows the raw single molecule imaging data averaged over 10 frames for data collected from the light sheet and epi using the same laser power and integration time. The SNR, defined as the ratio between the intensity of a single molecule event and of the background, is $\sim 2$ with epi, and $\sim 5$ with the light sheet illumination (Figure 3c). This high SNR improves the single-emitter fitting precision, and enhances
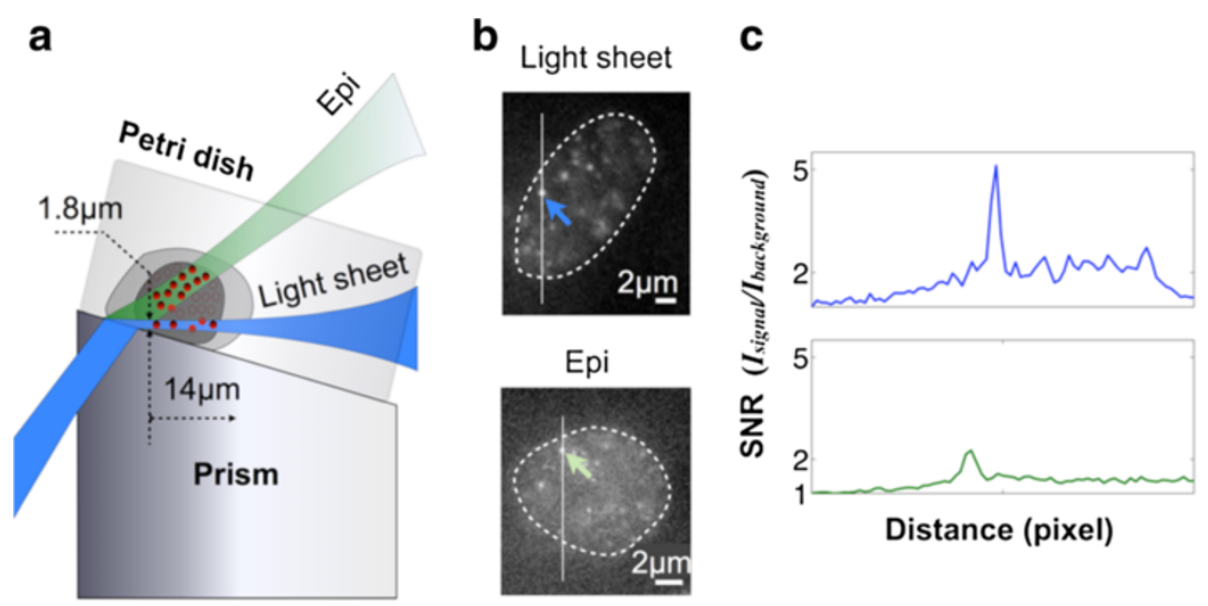

Figure 3 Light sheet illumination provides enhanced SNR for single-molecule imaging in the nucleus. (a) Schematic depiction of the light-sheet and epi-illumination. (b) Single-molecule images collected from light-sheet (top) and epi-illumination (bottom) of the nucleus of fixed Hues6 (hESC) cells expressing mEos3 on HP1a. (c) Cross profiles demonstrating the enhanced signal-to-noise ration of the single-molecule event from light-sheet (top) vs. epi-illumination (bottom). 
the efficiency of the reconstruction algorithm for resolving more events per frame.

\section{Super-resolution imaging using LSBM}

The Bayesian algorithm effectively resolves sub-diffraction cellular structures. We cross-compared the reconstructed structures between Bayesian and standard single-emitter fitting analysis using standard epi and light-sheet illumination for both thin and thick cell samples. Additional file 8: Figure S6 illustrates super-resolution heterochromatin foci structure in a fixed BJ fibroblast cell with epiillumination. Both the Bayesian and PALM approach revealed sub-diffraction clusters in each focus. Additional file 9: Figure S7 shows microtubule structures in a fixed H1 cell with light-sheet illumination. Because of the high background generated by out-of-focus excitation, the epiillumination with PALM analysis yielded relatively low resolution, while the Bayesian analysis with light-sheet illumination resolved much thinner fiber structures with higher resolution. We note that Bayesian analysis yields heterogeneous resolution, which is exaggerated in long and linear structures, such as microtubule fibers. Since the number of molecules in each frame is an estimated and non-negative variable in the Bayesian algorithm, its uncertainty leads to uneven thickness of the reconstructed fiber image. Nonetheless, the Bayesian method drastically outperforms other reconstruction programs when the same number of frames is used.

Albeit substantial reduced number of image frames, the Bayesian analysis can achieve comparable resolution to standard PALM/STORM techniques. Figure 4a,b show a conventional fluorescence image of microtubules in a fixed U2OS cell and a super-resolution image obtained from LSBM using 400 frames of the bleach-andblink data. The effective resolution is evaluated as the spacing between adjacent fibers that are not resolvable in the conventional image (i) and the cross-section of the tubulin fiber (ii) (Figure 4c). We show in Figure 4d that LSBM resolves $20-\mathrm{nm}$ microtubules and $30-\mathrm{nm}$ spacing between the fibers at the diverging fork. This 30$\mathrm{nm}$ lateral resolution is consistent with standard PALM/ STORM techniques reported in the literature (Huang et al. 2008). We further performed super-resolution imaging of tubulin fibers in thick $\mathrm{H} 1$ cells (an hESC cell line) and engaged mother-daughter centrioles in transgenic retinal pigmentation epithelial (RPE) cells expressing photoactivatable protein mEos 2 with a similar lateral resolution. These structures are located far deep into the cell for TIRF but can be readily reached by light sheet illumination. We show in Additional file 10: Figure S8 that these known structures are still resolvable by LSBM. More importantly, the Bayesian method drastically reduces the number of frames from tens of thousands to hundreds, significantly increasing the temporal resolution for live cell imaging.

LSBM imaging of heterochromatin in fixed and live hESCs We generated transgenic hESC cell lines expressing photoactivatable proteins mEos 2 or mEos3 on the heterochromatin protein HP1 $\alpha$ using lenti-viral infection. Known to be enriched at the heterochromatic sites and maintaining the stability of heterochromatin, HP1 $\alpha$ is regarded as a hallmark of heterochromatin (Cheutin et al. 2003). We performed imaging of the HP1 $\alpha$ distribution in two different hESC cell lines: H1 (for fixed-cell imaging) and Hues6 (for live-cell imaging). Figure 5a shows a representative frame-averaged conventional fluorescent image of HP1 $\alpha$ in a fixed hESC. One can observe a non-uniform distribution of $\mathrm{HP} 1 \alpha$ throughout the nucleus. However, the overall structure appears diffusive and featureless (Figure $5 \mathrm{a}$ without green dots). The lack of distinct heterochromatin domains appears to be consistent with the fact that hESCs have globally hyperactive transcriptional activities. Comparatively, the
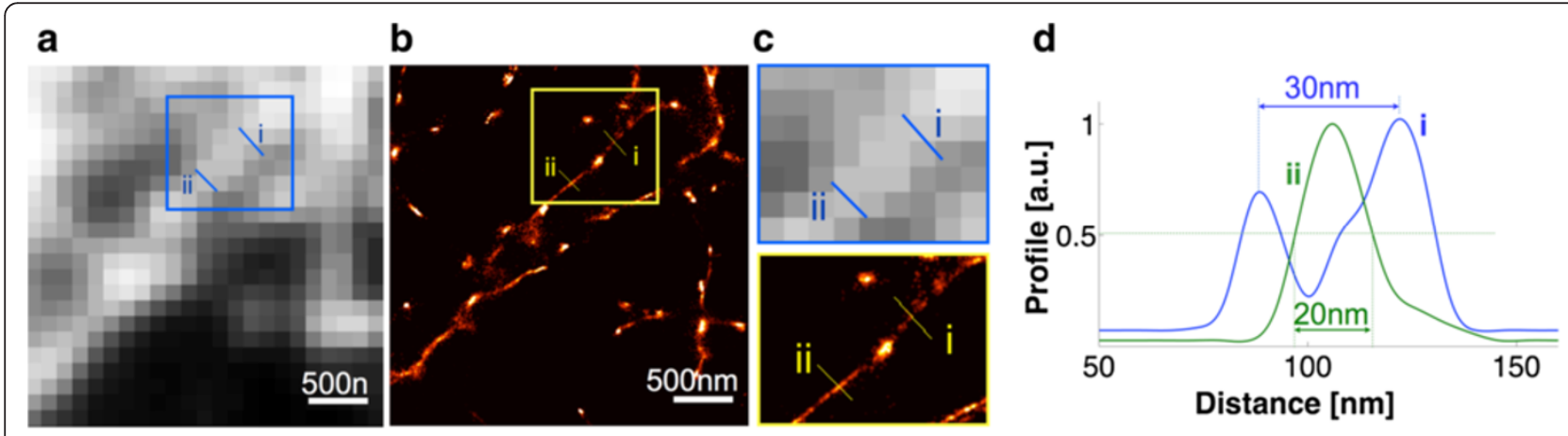

Figure 4 Bayesian reconstruction of microtubule fibers. (a) Conventional fluorescent image of the microtubules in a fixed U2OS cell. (b) Bayesian reconstruction of the superresolution image of (a) obtained from 642-nm light sheet illumination. (c) High-magnification view of the highlighted regions in (a) (upper panel) and (b) (lower panel). (d) Cross profile for fiber sections outlined in the lower panel in (c). The light sheet Bayesian microscope resolves 20-nm fibers separated by a distance of $30 \mathrm{~nm}$, demonstrating its lateral resolution of $20-30 \mathrm{~nm}$ with fixed samples. 

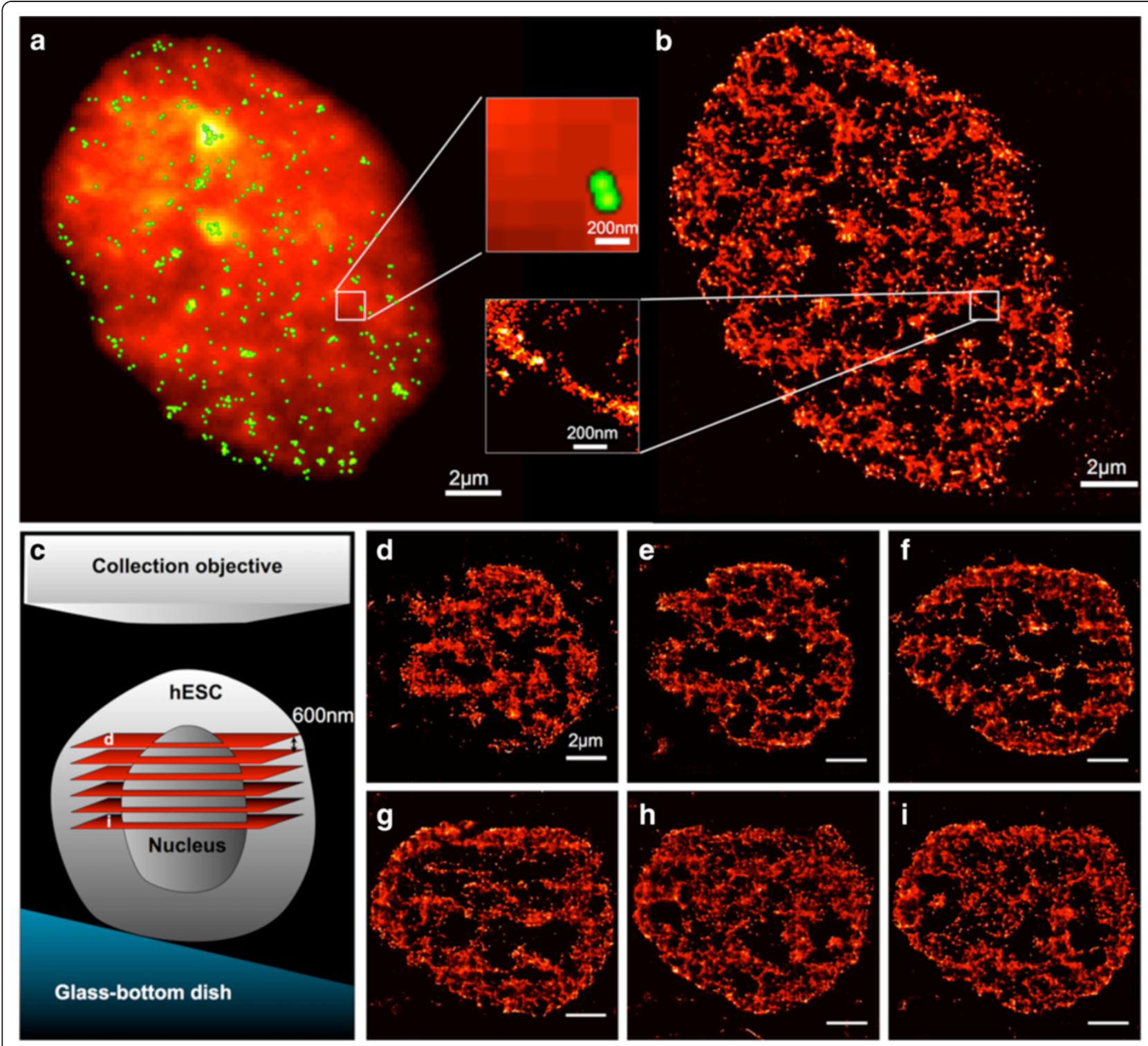

Figure 5 Light sheet Bayesian microscopy reveals the distribution of heterochromatin protein HP1a in an H1 cell. (a) Frame-averaged conventional fluorescent image of the distribution of mEos-tagged heterochromatic protein HP1a in a hESC (H1). Green dots are localizations generated by quickPALM using the same imaging data. (b) Bayesian reconstructed super-resolution image of the same region demonstrating distinct regions of HP1a. Inset panels contrast marked difference in resolution. (c) Schematic illustration of the light sheet sectioning at various depths of the nucleus. (d)-(i) Sections of super-resolution images of HP1a in the nucleus of an $\mathrm{H} 1$ cell. Conserved topographical structures were observed at different planes 600-nm apart in the axial direction.

super-resolution image generated from LSBM reveals distinct nanometer-scale features of heterochromatin in the entire cross-section of the nucleus (Figure 5b). The insets of Figure 5 illustrate a striking nanoscopic structure resolved by LSBM but not discernible in the conventional fluorescent image. We emphasize that the 3B algorithm is crucial for achieving the reported lateral resolution with only hundreds of frames. As a comparison, analysis using conventional single-molecule imaging methods, such as quickPALM (Henriques et al.
2010) or rapidSTORM (Wolter et al. 2012), with the same bleach-and-blink image frames, would only resolve sparse single-molecule events and fail to reconstruct the nanoscopic heterochromatin features (green dots in Figure 5a). The sparse localization is resulted from the program discarding most of the overlapping fluorophores.

The pattern of $\mathrm{HP} 1 \alpha$ distribution was consistently found at various depths of the nucleus (Figure 5c-i). By utilizing the slope of the sample placement, we achieved vertical sectioning by horizontally translating the sample 
via a piezoelectric stage (Online methods). As the lightsheet illumination moves from the top towards the bottom of the nucleus, the cross-section of the nucleus gradually increases. In addition to the mesh-like structures, one can observe void areas of heterochromatin near the center of the nucleus. These morphological features suggest organized heterochromatin compartments within the nucleus of the hESC.

With the enhanced temporal resolution, we demonstrate live-cell imaging of heterochromatin in hESCs using LSBM. We adapted the LSBM for live-cell imaging by maintaining the temperature of the glass prism and the imaging objective (Online methods). We continuously delivered activation power followed by bleach-andblink measurements, and reconstructed super-resolution images using 400 cropped frames captured within $2.3 \mathrm{~s}$ every minute for approximately 15 minutes (Online Methods). Time-lapsed conventional fluorescent images showed noticeable change to the morphology of the nucleus on the minute-scale (grey areas in Figure 6a). For instance, the length of the cross section changed from $21 \mu \mathrm{m}$ at $2 \mathrm{~s}$ to $18.5 \mathrm{um}$ at $542 \mathrm{~s}$. LSBM quickly resolved nanometer-scale heterochromatin structures within $2.3 \mathrm{~s}$, as demonstrated by red areas in Figure $6 \mathrm{a}$ as well as with greater details in Figure 6b,c. The lateral resolution allowed resolving 50-60 $\mathrm{nm}$ features and dynamic differences between time points. Fast data acquisition mitigates the adverse effect from instrument drift and cell motion. In the near future, we expect to couple the Bayesian algorithm with a fast sCMOS camera and
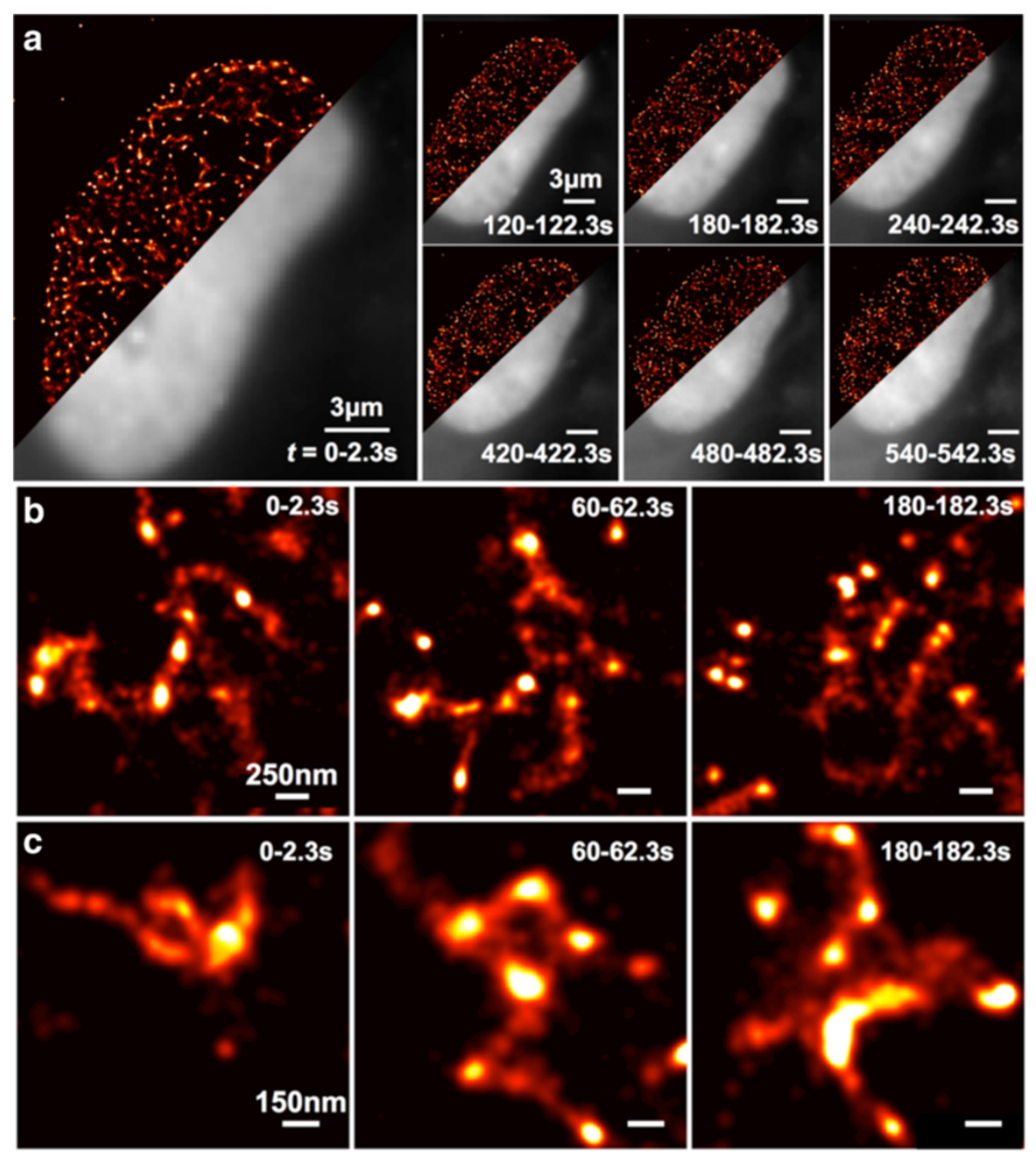

Figure 6 Super-resolution live cell imaging of the dynamics of HP1a in a Hues6 cell. (a) Light-sheet imaging of HP1a in a living Hues6 cell with 2.3-s data acquisitions. Grey areas denote the frame-average conventional fluorescent images and the red areas denote the superresolution images of the HP1a structure. Acquisition time points are as marked. (b)-(c), High-magnification views of the temporal dynamics of the HP1a distribution at two positions within the nucleus. 
achieve super-resolution imaging over 10 fps. (Huang et al. 2013).

\section{Conclusions}

Acquiring sub-cellular super-resolution images deep inside a living cell is one of the most challenging tasks in bioimaging. In this study, we designed LSBM and demonstrated its capability by visualizing the structure and dynamics of heterochromatin in fixed and live hESCs. With the fixed hESCs, we first demonstrated a mesh like heterochromatin structure in pluripotent stem cells. These structurally distinct organizations are also aligned with the topological domain hypothesis (Lieberman-Aiden et al. 2009; Dixon et al. 2012). The latest sequencing data suggest that mammalian genomes are organized into conserved topological chromatin-chromatin interaction domains for efficient large-scale gene expression regulation and constraining the spread of heterochromatin in different cell types (Dixon et al. 2012). The dynamic change of the nanoscopic structural features revealed in Figure 4 is a combination of the Brownian motion of the chromatin, the movement of heterochromatin in response to sub-cellular activities, and the cell motion (Dion \& Gasser 2013). LSBM provides a viable imaging tool to examine these dynamics, for the first time, with nanometer resolution. Further studies can be done to examine the heterochromatin structure and its dynamics in hESCs at different phases of the cell cycle and during their responses to different differentiation stimuli. Techniques can be applied to label a region of interest to help lock the imaging FOV in order to counter cell motion. The 2.3-s temporal resolution of LSBM is a unique capability enabled by the Bayesian algorithm. Importantly, using bright and fast-switching molecules, and increasing the frame rate of the camera, i.e. by using a cropped mode for a smaller FOV, can further increase the imaging speed to the sub-second level.

Our prism-coupled light sheet design provides high SNR for imaging thick hESCs and accommodates a highNA imaging objective, which is crucial for the photon collection efficiency for single-molecule super-resolution imaging. The majority of light sheet designs use an imaging lens with an NA of 0.8 or lower. However, a mere increase of 0.2 in NA from 0.8 to 1.0 increases the light collection efficiency by $32 \%$. Although we demonstrated single-molecule super-resolution imaging through $10-\mu \mathrm{m}$ thick nuclei in hESCs, the use of LSBM can be readily expanded for general biological samples more than a few micrometers thick, such as thin tissue slices, without modification. In fact, the robustness and ease of implementation is a significant advantage over other light-sheet techniques. Taken together, LSMB enables a wide array of in vivo studies from deep-cell imaging of sub-cellular structures to real-time probing of dynamic cellular processes with nanometer resolution.

\section{Additional files}

\begin{abstract}
Additional file 1: Figure S1. Laser scanning confocal microscope (LSM) sectioning images comparing the thickness of the nucleus between a somatic cell (BJ fibroblast) and a human embryonic stem cell (Hues6). The transgenic cells express HP1a-mEos3. While the nucleus of the BJ fibroblast is $\sim 3 \mu \mathrm{m}$ thick, the nucleus of an hESC is $3 \times$ thicker approaching $10 \mu \mathrm{m}$.

Additional file 2: Figure S2. a) Pictorial depiction of the light sheet Bayesian microscope. Top left panel: a photograph demonstrating the prism-coupled light sheet set-up; right panel: SolidWorks ${ }^{T M}$ rendering showing details of the prism placement with respect to the objectives. b) Schematic diagram of the optical setup. DM: dichroic mirror; BE: beam expander; CL: cylindrical lens; M: mirror; P: glass prism; GBD: glass bottom dish; TL: tube lens; F: emission and notch filters.
\end{abstract}

Additional file 3: Figure S3. Measured log-normal distribution of the brightness and size of single-molecule events with light-sheet illumination.

Additional file 4: Note S1. Implementation of the Bayesian bleach-andblink (3B) algorithm. Typically, a sample of interest is quickly bleached out using high laser intensity and the first 3-400 image frames are used for reconstruction. There are highly overlapping fluorophores at the beginning of the acquisition. After the initial quick bleaching, only a small subset of the molecules remains on in a stochastic fashion. Fitting the bleaching and blinking pattern allows extraction of considerably more single-molecule events from high-density areas. Thus, it achieves a comparable spatial resolution with fewer image frames. We parallelized the Bayesian calculation by dividing the 400-frame tiff file into smaller 10-by10-pixel sub-areas. Each adjacent sub-areas had a 5-pixel overlap in the horizontal and vertical direction. The spatial overlap reduces the edge artifact of the code when the boundary crosses through a high-density area. The parallelization allowed us to reconstruct larger image areas using cluster computing and super-computing resources. A minimum of 200 iterations were performed before the Bayesian algorithm was terminated.

Additional file 5: Figure S4. Electromagnetic simulation of the intensity of the light sheet at the prism/oil - water interface. Figures (a) (d) demonstrate the light sheet from being total-internal-reflected to being refracted into water. Incident and reflected Gaussian beams interfere and form interference pattern near the interface. This interference pattern can be clearly visible when imaging light sheet using fluorescent bead solution. The pattern also helps locating the interface when tuning the profile of the light sheet. The excitation wavelength is $561 \mathrm{~nm}$.

Additional file 6: Figure S5. Finite element simulation results showing the effect from index change of the medium on the light sheet. At the same incident angle of 27.5 degrees, and with the same incident light. (a)-(c) Finite element simulation showing the intensity of a focused Gaussian beam traveling from glass to a medium with a refractive index of $1.40,1.35$, and 1.30 , respectively. (d) Length and width of the light sheet as a function of the medium index. Light-sheet width was calculated from the FWHM of the intensity profile, light-sheet length was calculated from the glass-water interface along the light sheet to the point where its intensity drops by one half.

Additional file 7: Note S2. Light sheet characterization with different imaging media (refractive indices). For imaging fixed cells in PBS, which has a refractive index of 1.337 at $510 \mathrm{~nm}$ (Templer and Leatherbarrow 2002), close to the water index of 1.333, we do not expect significant change of the light sheet profile. For higher index changes, we characterized the effect of refractive index using a finite-element simulation. Additional file 6: Figures S5a-c plot the light sheet profile at three different indices. We expect the light sheet to bend up in high-index media, as described by the Snell's law. This will reduce the overlap between the light sheet and the imaging plane and thus decrease the FOV. In addition, index change affects the width and length of the light sheet. We measured width as the FWHM at the beam waist and length as the distance from the interface where light enters the medium to the point along its propagation where the intensity drops by a half. As the index decreases, the light sheet will tilt towards the interface and its length 
generally decreases. Its width, however, first increases (i.e., $n>1.38$ ) because a lower refractive index confined light in a lesser degree, and then decreases (i.e., $n<1.38$ ) because of the NA limited by the interface, i.e., intensity distribution is confined by the interface. In this particular simulation, broadening of $\sim 100 \mathrm{~nm}$ and elongation of $\sim 7 \mu \mathrm{m}$ are expected when the medium index changes from 1.33 to 1.40 . These changes increase the FOV and counter the reverse effect from the refractive angle. As indicated by the simulation, for live cell experiments, the incident angle has to compensate for the higher index from the cell medium.

Additional file 8: Figure S6. Validation of the $3 B$ code with PALM by imaging heterochromatin foci in a transgenic BJ fibroblast cell expressing mEos2 on HP1a. (a) Frame-averaged conventional image of the heterochromatin foci in a fixed BJ fibroblast cell. (b) High-magnification diffraction-limited view of the two foci. (c) Super-resolution images of the foci in (b) using a standard single-emitter fitting program. (d) Superresolution images of the foci in (b) using the Bayesian method. (e) Line profiles showing sub-diffraction features resolved by the two reconstruction methods.

Additional file 9: Figure S7. Super-resolution images of microtubule fibers with light sheet illumination reconstructed from (a) single molecule localizations using single-emitter fitting; (b) Bayesian analysis.

Additional file 10: Figure S8. Comparison of conventional fluorescence images and Bayesian superresolution images. (a-b) Conventional and Bayesian-reconstructed super-resolution images of the microtubule fibers in $\mathrm{H} 1 \mathrm{hESCs}$. (c) conventional fluorescent image of two adjacent centrioles in retinal pigmentation epithelium (RPE) cells (left) and Bayesian-reconstructed super-resolution image of the same region (right). Centrioles are tagged with mEos2 protein via trangenesis. While in the diffraction limited image, each centriole structure appears to be composed of two sub-units, the super-resolution image clearly demonstrates the mother and daughter centrioles that are still engaged.

\section{Abbreviations}

LSBM: Light-Sheet Bayesian Microscopy; 3B analysis: Bayesian bleach-andblink analysis; hESC: Human embryonic stem cell; SNR: Single-to-noise ratio; TIRF: Total internal reflection fluorescence microscopy; FOV: Field of view; SPIM: Selective-plane illumination microscopy; IML-SPIM: Individual molecule localization selective-plane illumination microscopy; iSPIM: Inverted selectiveplane illumination microscopy; HILO: Highly inclined and laminated optical sheet; AFM: Atomic force microscopy; EMCCD: Electron multiplying charge coupled device; RF: Radio frequency; FWHM: Full width at half maximum; NA: Numerical aperture; STORM: Stochastic optical reconstruction microscopy; PALM: Photoactivated localization microscopy; Amazon EC2: Amazon elastic compute cloud.

\section{Competing interests}

The authors declare no competing interests.

\section{Authors' contributions}

YSH, QZ, HC designed research. YSH performed experiments and data analysis. HC and YSH implemented the optical system. YSH and KT compiled and performed $3 \mathrm{~B}$ reconstruction. JF assisted with data visualization. QZ and KE generated the transgenic human embryonic stem cells. YSH and YL carried out immunostaining for STORM. YSH, QZ and HC prepared the manuscript. IMV assisted with the manuscript. All authors read and approved the final manuscript.

\section{Acknowledgements}

This work was funded by Cancer Center Core Grant (P30 CA014195-39), the Ray Thomas Edwards Foundation, and the Waitt Foundation. The computational resources at the National Energy Research Scientific Computing Center (NERSC) are supported by the Office of Science of the U.S. Department of Energy under Contract No. DE-AC02-05CH1 1231. Y.S.H. is supported by the California Institute of Regenerative Medicine postdoctoral training grant TG201158. Q.Z. was supported by Department of Defense Idea Expansion Award (W81XWH-10-1-0963). J.F is supported in part by NCI P30 CA014195-39 and NINDS P30 NS072031-01A1. I.M.V. is an American Cancer Society Professor of Molecular Biology, and holds the Irwin and Joan Jacobs Chair in Exemplary Life Science. We would like to thank Dr. Yongmin Liu for the assistance with
COMSOL simulations, Dr. Martin Hetzer for valuable comments on this manuscript, Marina Watanabe for providing human embryonic stem cells and Nina Tonnu for generating transgenic cells lines, Dr. Max Zimmerley for help preparing the manuscript, Dr. Prabuddha Sengupta at the NIH for providing the mEos3 construct, Dr. David Skinner and Dr. Jack Deslippe at the Lawrence Berkeley National Laboratory for help with compiling the Bayesian code on supercomputers at NERSC.

\section{Author details}

${ }^{1}$ Waitt Advanced Biophotonics Center, Salk Institute for Biological Studies, La Jolla, CA 92037, USA. ${ }^{2}$ Laboratory of Genetics, Salk Institute for Biological Studies, La Jolla, CA 92037, USA. ${ }^{3}$ Department of Bioengineering, University of California San Diego, La Jolla, CA 92093, USA. ${ }^{4}$ Current address:

Department of Cellular and Molecular Medicine, University of California San Diego, La Jolla, CA 92093, USA.

Received: 8 June 2013 Accepted: 20 October 2013

Published: 20 November 2013

\section{References}

Ahrens MB, Orger MB, Robson DN, Li JM, Keller PJ (2013) Whole-brain functional imaging at cellular resolution using light-sheet microscopy. Nat Methods 10 (5):413-420

Bates M, Huang B, Dempsey GT, Zhuang X (2007) Multicolor super-resolution imaging with photo-switchable fluorescent probes. Science 317(5845):1749-1753

Betzig E, Patterson GH, Sougrat R, Lindwasser OW, Olenych S, Bonifacino JS, Davidson MW, Lippincott-Schwartz J, Hess HF (2006) Imaging intracellular fluorescent proteins at nanometer resolution. Science 313(5793):1642-1645

Cella Zanacchi F, Lavagnino Z, Perrone Donnorso M, Del Bue A, Furia L, Faretta M, Diaspro A (2011) Live-cell 3D super-resolution imaging in thick biological samples. Nat Methods 8(12):1047-1049

Cheutin T, McNairn AJ, Jenuwein T, Gilbert DM, Singh PB, Misteli T (2003) Maintenance of stable heterochromatin domains by dynamic HP1 binding. Science 299(5607):721-725

Cox S, Rosten E, Monypenny J, Jovanovic-Talisman T, Burnette DT, LippincottSchwartz J, Jones GE, Heintzmann R (2012) Bayesian localization microscopy reveals nanoscale podosome dynamics. Nat Methods 9(2):195-200

Dion V, Gasser SM (2013) Chromatin movement in the maintenance of genome stability. Cell 152(6):1355-1364

Dixon JR, Selvaraj S, Yue F, Kim A, Li Y, Shen Y, Hu M, Liu JS, Ren B (2012) Topological domains in mammalian genomes identified by analysis of chromatin interactions. Nature 485(7398):376-380

Folling J, Belov V, Kunetsky R, Medda R, Schonle A, Egner A, Eggeling C, Bossi M, Hell SW (2007) Photochromic rhodamines provide nanoscopy with optical sectioning. Angewandte Chemie 46(33):6266-6270

Friedrich M, Nozadze R, Gan Q, Zelman-Femiak M, Ermolayev V, Wagner TU, Harms GS (2009) Detection of single quantum dots in model organisms with sheet illumination microscopy. Biochem Biophys Res Commun 390(3):722-727

Gebhardt JC, Suter DM, Roy R, Zhao ZW, Chapman AR, Basu S, Maniatis T, Xie XS (2013) Single-molecule imaging of transcription factor binding to DNA in live mammalian cells. Nat Methods 10(6):421-426

Henriques R, Lelek M, Fornasiero EF, Valtorta F, Zimmer C, Mhlanga MM (2010) QuickPALM: 3D real-time photoactivation nanoscopy image processing in ImageJ. Nat Methods 7(5):339-340

Hess ST, Girirajan TPK, Mason MD (2006) Ultra-high resolution imaging by fluorescence photoactivation localization microscopy. Biophys J 91(11):4258-4272

Hu YS, Nan X, Sengupta P, Lippincott-Schwartz J, Cang H (2013) Accelerating 3B single molecule super-resolution microscopy with cloud computing. Nat Methods 10(2):96-97

Huang B, Wang WQ, Bates M, Zhuang XW (2008) Three-dimensional superresolution imaging by stochastic optical reconstruction microscopy. Science 319(5864):810-813

Huang F, Hartwich TM, Rivera-Molina FE, Lin Y, Duim WC, Long JJ, Uchil PD, Myers JR, Baird MA, Mothes W, Davidson MW, Toomre D, Bewersdorf J (2013) Video-rate nanoscopy using SCMOS camera-specific single-molecule localization algorithms. Nat Methods 10(7):653-658

Keller PJ, Schmidt AD, Wittbrodt J, Stelzer EHK (2008) Reconstruction of Zebrafish early embryonic development by scanned light sheet microscopy. Science 322(5904):1065-1069 

Fast, high-contrast imaging of animal development with scanned light sheet-based structured-illumination microscopy. Nat Methods 7(8):637-642

Lidke KA (2012) Super resolution for common probes and common microscopes. Nat Methods 9(2):139-141

Lieberman-Aiden E, van Berkum NL, Williams L, Imakaev M, Ragoczy T, Telling A Amit I, Lajoie BR, Sabo PJ, Dorschner MO, Sandstrom R, Bernstein B, Bender MA, Groudine M, Gnirke A, Stamatoyannopoulos J, Mirny LA, Lander ES, Dekker J (2009) Comprehensive mapping of long-range interactions reveals folding principles of the human genome. Science 326(5950):289-293

Lippincott-Schwartz J, Patterson GH (2009) Photoactivatable fluorescent proteins for diffraction-limited and super-resolution imaging. Trends Cell Biol 19(11):555-565

Maison C, Almouzni G (2004) HP1 and the dynamics of heterochromatin maintenance. Nat Rev Mol Cell Biol 5(4):296-304

Moerner WE (2007) New directions in single-molecule imaging and analysis. Proc Natl Acad Sci U S A 104(31):12596-12602

Patterson G, Davidson M, Manley S, Lippincott-Schwartz J (2010) Superresolution Imaging using single-molecule localization. Annu Rev Phys Chem 61(61):345-367

Ritter JG, Veith R, Veenendaal A, Siebrasse JP, Kubitscheck U (2010) Light sheet microscopy for single molecule tracking in living tissue. PLoS One 5(7):e11639

Rosten E, Jones GE, Cox S (2013) ImageJ plug-in for Bayesian analysis of blinking and bleaching. Nat Methods 10(2):97-98

Rust MJ, Bates M, Zhuang XW (2006) Sub-diffraction-limit imaging by stochastic optical reconstruction microscopy (STORM). Nat Methods 3(10):793-795

Shroff H, Galbraith CG, Galbraith JA, Betzig E (2008) Live-cell photoactivated localization microscopy of nanoscale adhesion dynamics. Nat Methods 5(5):417-423

Siedentpf H, Zsigmondy R (1903) Über Sichtbarmachung und Groessenbestimmung ultramikroskopischer Teilchen, mit besonderer Anwendung auf Goldrubinglaesern. Annalen der Physik 10:1-39

Templer RH, Leatherbarrow R (2002) Biophysical Chemistry: Membranes and Proteins. The Royal Society of Chemistry, Cambridge:UK

Thomson JA, Itskovitz-Eldor J, Shapiro SS, Waknitz MA, Swiergiel JJ, Marshall VS, Jones JM (1998) Embryonic stem cell lines derived from human blastocysts. Science 282(5391):1145-1147

Tokunaga M, Imamoto N, Sakata-Sogawa K (2008) Highly inclined thin illumination enables clear single-molecule imaging in cells. Nat Methods 5(2):159-161

Tomer R, Khairy K, Amat F, Keller PJ (2012) Quantitative high-speed imaging of entire developing embryos with simultaneous multiview light-sheet microscopy. Nat Methods 9(7):755-763

Vaziri A, Tang J, Shroff H, Shank CV (2008) Multilayer three-dimensional super resolution imaging of thick biological samples. Proc Natl Acad Sci U S A 105(51):20221-20226

Wolter S, Loschberger A, Holm T, Aufmkolk S, Dabauvalle MC, van de Linde S, Sauer M (2012) rapidSTORM: accurate, fast open-source software for localization microscopy. Nat Methods 9(11):1040-1041

Woods NB, Parker AS, Moraghebi R, Lutz MK, Firth AL, Brennand KJ, Berggren WT, Raya A, Belmonte JCl, Gage FH, Verma IM (2011) Brief report: efficient generation of hematopoietic precursors and progenitors from human pluripotent stem cell lines. Stem Cells 29(7):1158-1164

Wu Y, Ghitani A, Christensen R, Santella A, Du Z, Rondeau G, Bao Z, Colon-Ramos D, Shroff H (2011) Inverted selective plane illumination microscopy (iSPIM) enables coupled cell identity lineaging and neurodevelopmental imaging in Caenorhabditis elegans. Proc Natl Acad Sci U S A 108(43):17708-17713

York AG, Ghitani A, Vaziri A, Davidson MW, Shroff H (2011) Confined activation and subdiffractive localization enables whole-cell PALM with genetically expressed probes. Nat Methods 8(4):327-333

Zwaka TP, Thomson JA (2003) Homologous recombination in human embryonic stem cells. Nat Biotechnol 21(3):319-321

doi:10.1186/2192-2853-2-7

Cite this article as: Hu et al:: Light-sheet Bayesian microscopy enables deep-cell super-resolution imaging of heterochromatin in live human embryonic stem cells. Optical Nanoscopy 2013 2:7.

\section{Submit your manuscript to a SpringerOpen ${ }^{\circ}$ journal and benefit from:}

- Convenient online submission

- Rigorous peer review

- Immediate publication on acceptance

- Open access: articles freely available online

- High visibility within the field

- Retaining the copyright to your article

Submit your next manuscript at $>$ springeropen.com 